\title{
EDITORIAL
}

\section{IN THE FEBRUARY, 2008 ISSUE OF CLINICS}

\author{
Mauricio Rocha-e-Silva, Editor
}

As Clinics begins its $4^{\text {th }}$ year of existence we are happy to announce our inclusion and indexing in the Journal of Citation Reports of the Institute for Scientific Information. This was the last significant indexing missing for Clinics; consequently, its first impact will be calculated early next year. Using JCR methodology, we appear to have closed 2007 with an extra-official impact factor above 0.800 , which would place us in good standing among the 23 ISI/ JCR indexed Brazilian journals, and approximately at the median of worldwide journals. We definitely hope to improve this position over the coming years.

In this issue we publish nine original papers on Clinical Science.

Bispo Júnior et al evaluated demographic and clinical variables of 978 operated patients, 109 of which presented at least two associated ligament injuries in the same knee and concluded that anterior cruciate ligament injuries are associated with sports and falls, while posterior cruciate ligament injuries and combined injuries relate to traffic accidents and direct traumas to the knee. A delay between primary ligament injuries and their reconstructions generates varus deformity of the affected knee.

Satomi et al examined endon samples from patients with and without posterior tibial tendon dysfunction and characterized the morphologic pattern and distribution of types I, III and V collagen in these dysfunctions: they found that control samples showed that type $\mathrm{V}$ deposited near the vessels only, while surgically obtained specimens displayed type $\mathrm{V}$ collagen surrounding other types of collagen fibers in thicker adventitial layers; type III collagen levels were also increased in pathological specimens; in contrast, type I collagen, which represents $95 \%$ of the total collagen in the normal tendon, was decreased in pathological specimens.

Arismendi-Morillo et al analyzed stroke and its relationship with cardiovascular and renal pathology in young

Hospital das Clínicas, Faculdade de Medicina da Universidade de São Paulo - São Paulo/SP, Brazil. mrsilva36@hcnet.usp.br adults through 52 clinical necropsies with diagnoses of stroke in patients aged 18 - 49 years, and found that hypertensive cardiopathy, occlusive atherosclerotic disease, chronic pyelonephritis and nephrosclerosis are among the pathophysiologycal mechanisms that apparently and eventually interact to induce a significant number of cases of stroke in young adults; a chronic systemic inflammatory state appears to be an important related condition because it possibly constitutes an accelerant of the pathophysiologycal process.

Lopes et al investigated the early adverse events related to the vaccine against influenza in 197 individuals and health care workers and conclude that the belief of part of the population that credits frequent and uncomfortable adverse events to the vaccine was not confirmed. Such subjective adverse events were more frequent in the health care workers, which can negatively influence the disclosure of the benefits of this vaccine due to their role as opinion makers.

Favano et al investigated whether supplementation of carbohydrate together with peptide glutamine increases exercise tolerance in 9 soccer players through a cardiopulmonary exercise test followed by a protocol simulating soccer game movements (intermittent exercise); carbohydrate with peptide glutamine or carbohydrate alone was administered randomly; the carbohydrate glutamine mixture was more efficient in increasing the distance covered and the length of time for which intermittent exercise was tolerated; it also reduced feelings of fatigue compared with carbohydrate alone.

Caruso et al performed a prospective observational study in 116 consecutive patients submitted to mechanical ventilation for $>72$ hours to determine predictors of maximal inspiratory pressure and found that $40 \%$ of the mechanically ventilated patients had a decreased maximal inspiratory pressure during mechanical ventilation, which was independently associated with deeper levels of sedation. There was no relationship between the evolution of maximal inspiratory pressure and the muscular strength of the limb. 
Cunha et al investigated the presence of growth hormone receptor in plexiform neurofibromas of neurofibromatosis type 1 of 5 patients and found that 4 out of the 5 plexiform neurofibromas were immunopositive for growth hormone receptor, suggesting that growth hormone may influence the development of plexiform neurofibromas in patients with neurofibromatosis type 1 .

Pierin et al compared blood pressure measurements taken in 44 patients by physicians, nurses, and the patients themselves in the physician's office, in the ambulatory and at home; the pressure measurement most similar to home (by healthcare giver) and ambulatory readings was pressure measured by the patient, and that home and ambulatory readings had good prognostic value relative to "office measurements".

Carvalho et al evaluated clinical indicators of child mental development of 737 infants in the capitals of nine Brazilian states to assess the influence regional cultural factors; finding that local habits and culture did not affect the results.

We publish eight original articles on Basic Science

Silveira et al evaluated the correlation between intrasac pressure measurements of a pressure sensor and an angiographic catheter during endovascular repair of abdominal aortic aneurysm in 25 patients; simultaneous readings of systolic, diastolic, mean, and pulse pressure within the aneurysm sac were obtained from catheter and sensor, before and after sac exclusion and conclude that the excellent agreement between intrasac readings by catheter and sensor justifies use of the latter for detection of post-exclusion aneursysm pressure.

Orra et al measured the pulsatility of 5 human aortic aneurysms obtained during necropsy before and after complete exclusion with an endograft and found that the amplitude of pulsation dropped following exclusion, but not significantly and never to zero; they conclude that pulsation is transmitted to the aneurysm wall even in the absence of endoleak, and should not be interpreted as procedural failure.

Sasaki et al experimentally compared single bundle versus anatomical double bundle techniques for anterior cruciate ligament reconstruction in 9 pairs of cadaver knees and conclude that the 2 types of reconstruction of the anterior cruciate ligament with bone-patellar tendon-bone graft have similar biomechanical behavior with regard to anterior tibial dislocation, rigidity, and passive internal tibial rotation.

Oliveira-Júnior et al described the effects of pentoxifylline on tnf-alpha and lung histophatology in $\mathrm{HCl}$ induced lung injury in 20 male, adult Wistar-EPM-1 anesthetized rats and concluded that pretreatment with pentoxifylline improves oxygenation, reduces TNF- \pm concentration, and increases the concentration of corticosteroid in BAL upon lung lesion induced by $\mathrm{HCl}$.

Nayak et al studied variations of Radial Wrist Extensor Muscles in 48 (24 on the right side and 24 on left side) male upper limb forearms of human cadavers; all encountered variations are described, as information for surgeons about the different varieties of additional radial wrist extensors and the frequency of their occurrence;

Emre at al described the properties of sodium nitroprusside as a nitric oxide donor in a rat intestinal ischemiareperfusion model to investigate its efficacy in the reduction of the intestinal ischemia-reperfusion injury as a nitric oxide donor after intraperitoneal administration; significant tissue injury in histological sections and an increase in tissue levels of Malonyldialdehyde was detected; sodium nitroprusside alone and in combination with L-NAME reduced injury scores.

Pinto et al performed an anatomical study on the use of a galeal flap based on superficial temporal vessels for oral cavity and pharynx reconstruction through 40 dissections in 34 human cadavers and concluded that this galeal flap based on the superficial temporal vessels presents favorable anatomical characteristics for oral cavity and pharyngeal reconstruction.

Barros Filho and Molina evaluated the sensitivity and reproducibility of the Basso, Beattie, Bresnahan functional scale in the assessment of the locomotor capacity of rats after spinal cord injury and found that the scale has high reproducibility and satisfactory sensitivity for mild injuries, satisfactory reproducibility and non-satisfactory sensitivity for moderate injuries, but reduced reproducibility and nonsatisfactory sensitivity for severe injuries.

We also publish 2 review articles on "Sepsis: from bench to bed side", by Silva and Figueiredo, and on NonHeart Beating Donors, by Chaib, and 5 case reports. 